\title{
T-semimaximal submodules
}

\author{
Farhan Dakhil Shyaa \\ Department of Mathematics, University of Al-Qadsiyah, Al-Qadsiyah, Iraq \\ farhan.shyaa@qu.edu.iq farhan_math1@yahoo.co.uk
}

Recived : $14 \backslash 9 \backslash 2017$

Revised : 8\10\2017

Accepted : 16\10\2017

\begin{abstract}
In this paper, we define and study the notions of t-semimaximal submodule as a generalization of semimaximal submodule. We provided many properties and characterizations of this concept are provided.
\end{abstract}

Key words: maximal submodule, semimaximal submodule, t-semimaximal submodule and t-semisimple modules.

\section{Mathematics Subject Classification 2010:16 D10, 16D60, 16 D80.}

\section{Introduction}

Throughout this paper $R$ is a ring with unity and $M$ unitary a right $R$-module. The second singular (or Goldi torsion) of $M$ is denoted by $Z_{2}(M)$ and defined as $\mathrm{Z}\left({ }^{M} / \mathrm{Z}(\mathrm{M})\right)={ }^{\mathrm{Z}(\mathrm{M})} / \mathrm{Z}_{2}(\mathrm{M})$ where $\mathrm{Z}(\mathrm{M})$ is

the singular submodule of $M[5]$. A module $M$ is called $\mathrm{Z}_{2}$-torsion if $Z_{2}(M)=M$. A submodule $A$ of an $R$-module $M$ is said to be essential in $M$ (denoted by $A \leq_{\text {ess }} M$ ), if $\mathrm{A} \cap \mathrm{W} \neq(0)$ for every non-zero submodule $\mathrm{W}$ of $\mathrm{M}[7]$.

The concept of t-essential submodules is introduced as a generalizations of essential submodules [2]. A submodule $N$ of $M$ is said to be t-essential in $M$ (denoted by $\left(N \leq_{\text {tes }} M\right)$ if for every submodule $B$ of $M, N \cap B \leq$ $Z_{2}(M)$ implies that $B \leq Z_{2}(M)$. A submodule $N$ of a module $M$ is called small in $M$ and denoted by $N \ll M$ if for every $K \leq M$ the equality $M=N+K \quad$ implies $M=K$. A module $M$ is called hollow if every proper submodule of $M$ is small in $M$ "[10].
Asgari and Haghany in [3] introduced the concept of $\mathrm{t}$-semisimple modules and $\mathrm{t}$ semisimple rings; A module $M$ is called tsemisimple if every submodule $N$ of $M$ contains a direct summand $K$ of $M$ such that $K$ is t-essential in $N$. A submodule $N$ of a module $M$ is called semimaximal if $M / N$ is a semisimple module [9].

In this paper, we introduce a generalization of semimaximal submodule, namely tsemimaxmal. A submodule $N$ of a module $M$ is called t-semimaxmal if $M / N$ is t-semisimple module. This paper consists of two sections, in section two of this paper, we define and study the concept of t- maximal submodules and give some properties and charerizations of it.

Proposition (1.1)[2]:" The following statements are equivalent for a submodule A of an R-module $\mathrm{M}$ :

(1) $A$ is t-essential in $\mathrm{M}$.

(2) $\quad\left(A+Z_{2}(\mathrm{M})\right) / \mathrm{Z}_{2}(\mathrm{M})$ is essential in $M / \mathrm{Z}_{2}(M)$

(3) $\quad A+Z_{2}(M)$ is essential in $\mathrm{M}$;

(4) $M /_{A}$ is $Z_{2}$-torsion [3]". 
Corollary (1.2) [3]:" Let $M$ be a tsemisimple module. Then:

(1) Every submodule of $\mathrm{M}$ is t-semisimple.

(2) Every homomorphic image of $\mathrm{M}$ is $\mathrm{t}$ semisimple".

Corollary (1.3) [3]: "A module $M$ is tsemisimple if and only if $M$ has no proper tessential submodule which contains $Z_{2}(M)^{\prime \prime}$.

Corollary (1.4) [3]: Every direct sum of tsemisimple modules is t-semisimple.

\section{2. t-semimaxmal submodules}

in this section, we will introduce and study the concept of t-semimaximal submodule

Definition (2.1): A submodule $N$ of module $M$ is called t-semimaximal if $M /{ }_{N}$ is a t-semisimple module.

Proposition (2.2): Let $M$ be an $R$-module. $Z_{2}(M)$ is semimaximal submodule of $M$ if and only if $Z_{2}(M)$ is t-semimaximal submodule of M.

Proof: $\Rightarrow$ It is clear.

$\Leftarrow$ Since $Z_{2}(M)$ is t-semimaximal submodule of $M$, then $M / Z_{2}(M)$ is a t-semisimple module. Hence $\stackrel{M}{ } / Z_{2}(M) / Z_{2}\left(M / Z_{2}(M)\right)^{\text {is }}$ semisimple by [3, Theorem 2.3], but $Z_{2}\left(M / Z_{2}(M)\right)=(0)$. Hence $M / Z_{2}(M)$ is semisimple module. Thus $Z_{2}(M)$ is semimaximal submodule of $M$.

\section{Remarks and Examples (2.3):}

It is clear that every semimaxmal submodule of a right $R$-module is tsemimaxmal submodule but not conversely, for example: $4 Z$ is a tsemimaximal submodule of $Z$ as $Z$ module ( because $Z / 4 Z$ is $\mathrm{t}$ semisimple $Z$-module [3])

(2) Every t-essential (or essential) submodule $N$ of $M$ is t-semimaxmal (by [3, Example 2.2(i)])

(3) Let $N \leq M$ and $W$ be the complement of $N$, then $N \oplus W$ is tsemimaxmal of $M$.

(4) Let $N \leq W \leq M$ and $N$ be a tsemimaxmal submodule, then $W$ is a t-semimaxmal submodule of $M$.
Proof: Let $f: M /{ }_{N} \mapsto M /{ }_{W}$ defined by $f(m+N)=m+W$, for all $m \in M$.

It is clear that $f$ is a well-defined and epimorphism. Since $M / N$ is t-semisimple it follows from Hence $\frac{M}{W}$ is t-semisimple by Corollary $1.2(2)$ that $M / W$ is t-semisimple and hence $W$ is a t-semimaxmal submodule of $M$.

(5) If $N$ is t-semimaximal of $M$ and $N \leq K \leq M$, then $N$ is tsemimaximal of $K$.

Proof: Since $N$ is t-semimaxmal of $M$ it follows that $M /_{N}$ is t-semisimple. But $K /{ }_{N} \leq M /{ }_{N}$, hence by Corollary $1.2(1) \mathrm{K} /{ }_{N}$ is t-semisimple. Thus $N$ is t-semimaxmal of $K$.

(6) Let $\left\{M_{i}, i \in I\right\}$ be a family of $R$ modules and let $=\bigoplus_{i \in I} M_{i}$. If $A_{i}$ is tsemimaximal of $M_{i}$, then $\bigoplus_{i \in I} A_{i}$ is tsemimaxmal of $\bigoplus_{i \in I} M_{i}$.

Proof: Since $A_{i}$ is t-semimaxmal of $M_{i}$, it follows that $M_{i} /_{A_{i}}$ is t-semisimple and hence $\oplus_{i}{ }^{M_{i}} /_{A_{i}}$ is t-semisimple by Corollary 1.4. Thus $\bigoplus_{i \in I} A_{i}$ t-semimaxmal of $\bigoplus_{i \in I} M_{i}$.

(7) Let $\leq K \leq M$. Then $K \mathrm{a}$ tsemimaximal submodule if and only if of $M, K / B$ is a t-semimaxmal submodule of $M /_{B}$.

Proof: $\Rightarrow$ Since $K$ is a t-semimaxmal submodule of $M$, it follows that $M / K_{K}$ tsemisimple. But $\frac{M / B}{K / B} \simeq M / K$, it follows that $\frac{M / B}{K / B}$ is a t-semisimple module and hence $K / B$ is a t-semimaximal submodule of $M / B$. $\Leftarrow$ By similarly way of first direction.

(8) $\operatorname{Rad}(M)$ is t-semimaxmal submodule of $M$ if and only if $M=M_{1} \oplus M_{2}$ such that $M_{1}$ is semisimple and $\operatorname{Rad} M \leq_{\text {tes }} M_{2}$ [3, Proposition 2.10].

(9) If (0) is a t-semimaxmal submodule of a module $M$ then $N$ is tsemimaxiaml, for each non-zero submodule $N$ of $M$. 
Proof: suppose that (0) is t-semimaxmal submodule of a module $M$, thus $M /(0) \simeq$ $M$ is t-semisimple. Hence $M / N$ is tsemisimple by [3, Corollary 2.4(2)]. Thus $N$ is t-semimaxmal.

(10) If $N$ is a nonzero t-semimaxmal submodule (0) need not be $\mathrm{t}-$ semimaxmal, for example: $6 Z$ in $Z$ module is t-semimaxmal. But $(0)$ is not t-semimaxmal since $Z /_{(0)} \simeq Z$ is not t-semisimple

(11) $\quad M$ is t-semisimple $R$-module if and only if $M$ is t-semisimple $R / \operatorname{ann}(M)(0)$-module.

Proof: Since every submodule of $M R$ module if and only if every submodule of $M$ $R / \operatorname{ann}(M)$ module [10].

Proposition (2.4): Every submodule of tsemisimple $R$-module is t-semimaxmal submodule.

Proof: Let $U \leq M$ and $\pi: M \mapsto M / U$ be the natural epiomorphism. Hence $M / U$ is tsemisimple by Corollary 1.2(2). Thus $U$ is tsemimaxmal.

Proposition (2.5): The intersection of any two t-semimaximal submodules of an $R$ module is t-semimaximal submodule.

Proof: Let $U_{1}, U_{2}$ be two t-semimaximal submodules of $M$. Thus $M / U_{1}$ and $M / U_{2}$ are tsemisimple modules and hence $M / U_{1} \oplus^{M} / U_{2}$ is t-semisimple by Corollary 1.4. Since $M / U_{1} \cap U_{2}$ is an isomorphism to a submodule of $M / U_{1} \oplus^{M} / U_{2}$ it follows that $M / U_{1} \cap U_{2}$ is t-semisimple. Thus $U_{1} \cap U_{2}$ is a t-semimaxmal submodule of $M$.

Proposition (2.6): Let $U_{1}$ be a tsemimaximal submodule of an R-module $\mathrm{M}_{1}$ and $\mathrm{U}_{2}$ be a t-semimaxmal submodule of an R-module $\mathrm{M}_{2}$. Then $\mathrm{U}_{1} \oplus \mathrm{U}_{2}$ is a $\mathrm{t}$ semimaximal submodule of $\mathrm{M}_{1} \oplus \mathrm{M}_{2}$.

Proof: By hypothesis, $M / U_{1}$ and $M / U_{2}$ are tsemisimple $R$-module and hence from Corollary 1.4 we have that $M / U_{1} \oplus^{M} / U_{2}$ is tsemisimple Since $\quad M_{1} \oplus M_{2} / U_{1} \oplus U_{2} \simeq$ $M / U_{1} \oplus M / U_{2}$. It follows that ${ }^{M} \oplus M_{2} / U_{1} \oplus U_{2}$ is t-semisimple and hence $U_{1} \oplus U_{2}$ is tsemimaximal in $M_{1} \oplus M_{2}$.
Proposition (2.7): Let $M$ be an $R$-module and $N \leq M$. Then $N$ is a t-semimaxmal if and only if $M / W$ is semisimple, for each t-closed submodule $W$ of $M$ and $W \supseteq N$.

Proof: $\Rightarrow$ Let $W$ be a t-closed submodule of $M$ with $W \supseteq N$. Hence $M / W$ is a t-closed in $M /{ }_{N}$ by [4, Lemma 2.5]. But $N$ is a tsemimaxmal by hypothesis, so $M / N$ is tsemisimple. Then by [3, Corollary 2.17], $M / N / W /_{N}$ is semisimple and hence $\frac{M}{W}$ is semisimple.

$\Leftarrow$ To prove $N$ is a t-semimaximal submodule of $M$. Let $C / N$ be a t-closed in $M / N$, hence $C$ is a t-closed of $M$, and $C \supseteq N$. So that $M / C$ is semisimple by hypothesis, but $M /_{C} \simeq$ $M / N / C{ }_{N}$ so that $M / N / C_{N}$ is semisimple for each t-closed submodule $C /{ }_{N}$ of $M /{ }_{N}$, which implies $M /{ }_{N}$ is t-semisimple by [3, Corollary 2.17]. Thus $N$ is t-semimaxmal submodule of $M$.

Proposition (2.8): If $\operatorname{Rad}(M)$ is a tsemimaxmal and $M$ is hollow then $M / \operatorname{Rad}(M)$ is $Z_{2}$-torsion.

Proof: Since $\operatorname{Rad}(M)$ is t-semimaxmal, $M / \operatorname{Rad}(M)$ is t-semisimple. By [3, Proposition 2.10] we have that $M=M_{1} \oplus M_{2}$ where $M_{1}$ is semisimple and $\operatorname{Rad}(M) \leq_{\text {tes }} M_{2}$. Let $A \ll M$, then $A \leq \operatorname{Rad}(M) \leq_{\text {tes }} M_{2}$, so if $M$ is hollow, every submodule of $M$ contain in $M_{2}$. Hence $M=M_{2}$ and thus $M / \operatorname{Rad}(M)$ is $Z_{2}$-torsion.

Proposition (2.9): Let $N \leq M$. If $\left(N:_{R} M\right)$ is t-semimaxmal ideal in $R$, then $N$ is tsemimaximal.

Proof: Since $\left(N:_{R} M\right)$ is t-semimaxmal ideal in $R, R /(N: M)$ is a t-semisimple $R$-module. Since $M / N$ is an $R$-module, $\frac{M}{N}$ is an $\bar{R}$-module where $(\bar{R}=R / \operatorname{ann}(M / N)$ that is $M / N$ is an $R /\left(N:_{R} M\right)^{-m o d u l e . ~ H e n c e ~} M / N$ is a tsemisimple $\bar{R}$-module. Hence $M / N$ is a tsemisimple $R$-module (by Remarks and Examples 2.3(13)). Thus $N$ is t-semimaxaml.

Remark (2.10): If $R$ is t-semisimple ring and $M$ is an $R$-module, then every submodule of $M$ is t-semimaxaml. 
Proof: Since $R$ is a t-semisimple, every $R$ module $M$ is t-semisimple[3, Theorem 3.2]. Hence by Proposition 2.3 every submodule of $M$ is t-semimaxiaml.

Proposition (2.11): Let $N \leq M$. Then $N$ is a t-semimaxiamal submodule in $M$ if and only if for each submodule $A$ of $M$ with $A \supseteq N$, there exist $K, K^{\prime} \leq N$ such that $A=K+K^{\prime}$ and $M=K+L$ for some $L \leq M$ and $N \leq_{\text {tes }} K^{\prime}, K \cap L=N, K \cap K^{\prime}=N$.

Proof: $\Rightarrow$ Let $N$ be a t-semimaxmal submodule in $M$, then $M / N$ is t-semisimple. For each $A \supseteq N, \frac{A}{N} \leq \frac{M}{N}$. Hence by $[3$, Proposition 2.13(3)] $A /{ }_{N}=K /{ }_{N} \oplus^{K^{\prime}} /{ }_{N}$ for each $K, K^{\prime} \leq M$ with $K /{ }_{N} \leq \oplus M /{ }_{N}$ and $K^{\prime} /_{N}$ is $Z_{2}$-torsion. Hence $N \leq_{\text {tes }} K^{\prime}$ by Proposition 1.1. $K /{ }_{N} \leq^{\oplus M} /{ }_{N}$, then $K /{ }_{N} \oplus L /{ }_{N}=M /{ }_{N}$ for some $L \leq M$ with $N \leq L$, then $K+L=M$ with $K \cap L=N$.

$\Leftarrow$ For any $A /{ }_{N} \leq M / N$. As $A=K+$ $K^{\prime}, K \cap K^{\prime}=N$, then $A /{ }_{N}=K /_{N} \oplus K^{\prime} /_{N}$. Also, $K+L=M, K \cap L=N$, then $K /{ }_{N} \oplus^{L} /{ }_{N}=M / N$, so $K /{ }_{N} \leq^{\oplus M} /{ }_{N}$ But $N \leq_{\text {tes }} K^{\prime}$ implies $K^{\prime} /_{N}$ is $Z_{2}$-torsion. Hence $A /{ }_{N}=K /{ }_{N} \oplus K^{\prime} /{ }_{N}$ with $K /{ }_{N} \leq M /{ }_{N}$ and $K^{\prime} /{ }_{N}$ is $Z_{2}$-torsion implies $M / N$ is t-semisimple by [3, Proposition 2.13(3)]. Thus $N$ is t-semimaxmal submodule in $M$.

Proposition (2.12): An $R$-module $M$ is tsemisimple if and only if $\forall N \leq M, N+$ $Z_{2}(M)$ is semimaxmal.

Proof: $\Rightarrow$ Suppose that $M$ is t-semisimple, then $N+Z_{2}(M)$ is closed in $M, \forall N \leq M$ by [3,Corollary 2.8]. But $N+Z_{2}(M)$ contains $Z_{2}(M)$, so $N+Z_{2}(M)$ is t-closed [2,Proposition 2.6(4)]. Hence by [3, Corollary 2.17], $M / N+Z_{2}(M)$ is semisimple.

$\Leftarrow$ Since $\quad \forall \quad N \leq M, N+Z_{2}(M)$ is semimaxmal, so that $M / N+Z_{2}(M)$ is semisimple. Hence $M / N+Z_{2}(M)$ is semisimple ( if $N=0$ ). This implies $M$ is tsemisimple [3, Theorem 2.3].

\section{References}

[1] Anderson, F. W. and Fuller K. R. (1992). Rings and Categories of Modules, Second Edition, Graduate Texts in Math., Vo1.13, Springer-Verlag, Berlin-Heidelberg-New York.

[2] Asgari, Sh., Haghany, A. (2010). Densely co-Hopfian modules. Journal of Algebra and Its Aplications 9(6):989-1000.

[3] Asgari, Sh., Haghany, A. and Tolooei Y. (2013). T-semisimple modules and Tsemisimple rings comm. Algebra ,41(5):18821902.

[4] Asgari, Sh., Haghany, A. (2011). tExtending modules and t-Baer modules, Comm.Algebra, 39(5):1605-1623.

[5] Chatters, A. W. and Khuri, S. M. (1980). Endomorphism rings of modules over nonsingular CS rings, J. London Math. Soc. 21:434-444.

[6] Chen, J., Ding, N. and Yousif, M. F. (2004). On Noetherian rings with essential socle, J. Aust. Math. Soc., 76:39-49.

[7] Clark, J., Lomp, C., Vanaja N., Wisbauer, R. (2006). Lifting Modules. Frontiers in Mathematics, Birkh $\square$ auser Verlag, Basel.

[8] Dung, N. V., Huynh, D. V., Smith, P. F, Wisbauer, R. (1994). Extending Modules. Pitman Research Notes in Mathematics 313, Longman, Harlow.

[9] Hatem Yahya .(2007). Semimaximal submoduless, Ph.D. Thesis, College of Education Ibn Al-Haitham, University of Baghdad.

[10] Kasch F. Modules and Rings (1982), Acad. Press, London. 


\title{
المقاسات الجزئيه العظمئ من النمط T T
}

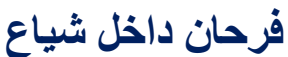 \\ جامعة القادسية / كلية التربية / قنم الرياضيات
}

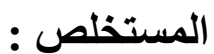

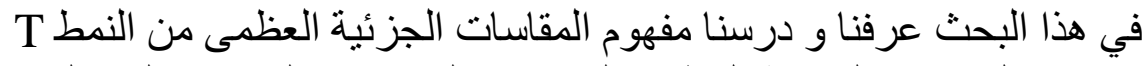
كتعميم المقاسات الجزئية العظمى العديد من الخواص والجزئن المميزات لهذا المفهوم برهنت. 